\title{
Excited-state regulation in eco-friendly ZnSeTe-based quantum dots by cooling engineering
}

\author{
Baoqiang $\mathrm{Wu}^{1 \dagger}$, Shuangyi $\mathrm{Zhao}^{2 \dagger}$, Mingshui Zhang ${ }^{1 \dagger}$, Zhigao Huang ${ }^{1}$, Chen $\mathrm{Chen}^{3}$, Zhigang Zang ${ }^{2}$ and \\ Yue Wang ${ }^{1 *}$
}

\begin{abstract}
The production of high-quality eco-friendly quantum dots (QDs) is challenging because of the efficient yet elusive nonradiative recombination within. This study examined the effects of cooling engineering on regulating the excited states to realize high-quality ZnSeTe core-shell QDs. The presence of ultrafast hot-carrier trapping and band-edge carrier trapping is responsible for the poor emission efficiency in ZnSeTe QDs. The above processes can be suppressed simultaneously by engineering the cooling process, and the underlying mechanisms are interrogated by combined electronic and spectroscopic characterization. The engineered ZnSeTe QDs exhibited record-high efficiency (>90\%) and stability that were comparable to those of the canonical CdSe QDs. Leveraging on the achievement, the ZnSeTe QD-based white light-emitting diodes (WLEDs) showed excellent optical performance, including a high color-rendering index of 80 and an appropriate correlated color temperature of 7391 K. Furthermore, the WLEDs could serve as light sources in ecofriendly visible light communication. These results highlight the feasibility of eco-friendly QDs for practical applications without environmental hazards.
\end{abstract}

Keywords: eco-friendly quantum dot, ZnSeTe, carrier dynamics, white light emission, visible light communication

\section{INTRODUCTION}

Colloidal semiconductor quantum dots (QDs) have been used widely as light emitters in various optoelectronic devices, such as light-emitting diodes (LEDs) and liquid crystal display backlight converters [1-6]. The tunable emission accomplished by the quantum-size-effect and composition modulation highlights the potential of QDs as promising luminescent materials [7,8]. By virtue of the well-established solution-phase approaches and the high photoluminescence quantum yield (PLQY), the classical CdSe-based QDs and the emerging $\operatorname{APbX}_{3}(\mathrm{~A}=\mathrm{FA}, \mathrm{MA}$ and Cs; $\mathrm{X}=\mathrm{Cl}, \mathrm{Br}$ and I) perovskite QDs have been widely investigated and adopted in light-emitting devices [9-12]. However, the involvement of heavy-metal elements including $\mathrm{Cd}$ and $\mathrm{Pb}$ may hinder their commercial applications. On basis of this, heavymetal-free QDs, such as InP, $\mathrm{Ag}_{2} \mathrm{Se}$, and $\mathrm{AgInSe}_{2}$, have been explored to substitute for toxic materials [13-16]. In this regard, InP-based QDs have reached a high PLQY of $\sim 100 \%$, but this has only been realized in the long visible region. Bulk InP has a bandgap of $1.35 \mathrm{eV}$, meaning that the core needs to have a small size to achieve blue-to-green emission. In this case, more carrier trapping defects would be formed, thus hindering the achievement of high PLQY in the short visible range, especially in the blue range [3,17]. Thus far, despite the impressive progress, the acquisition of eco-friendly QDs with high PLQY comparable to $\mathrm{Cd}$ - and $\mathrm{Pb}$-based QDs, particularly in the blue-to-green spectral range, is still challenging.

Recently, heavy-metal-free ZnSeTe-based QDs have been reported to show tunable blue-to-green emission by simply changing the $\mathrm{Se} / \mathrm{Te}$ ratio, making them promising for optoelectronic devices. However, these ZnSeTe QDs suffer from poor PLQY because of the presence of rich carrier traps $[18,19]$. The breakthroughs in the past few years using a multi-shell structure have improved the PLQY of blue- and green-emitting ZnSeTebased QDs by up to $80 \%$ and $68 \%$, respectively [20-22]. Nevertheless, these values are still far below those of the traditional QDs. Moreover, ZnSeTe-based QDs are prone to degradation compared with well-established CdSe QDs, further restricting their practical applications. Therefore, the development of high-quality ZnSeTe QDs is essential for making full use of their benefits.

The poor PLQY in ZnSeTe QDs lies in the efficient yet elusive nonradiative recombination of the excited state. In traditional CdSe QDs, the nonradiative recombination processes are well understood and can be controlled using synthetic methods $[23,24]$, whereas it remains less explored in ZnSeTe QDs. Generally, the growth of QDs follows the well-established LaMer model, which includes three processes: nucleation, crystal growth, and reaction termination [25-27]. From the experience of improving the PLQY of traditional QDs, synthetic optimization should focus on the first two processes. However, little progress has been made despite the attempts to optimize ZnSeTe-based QDs, such as changes in the composition of reactants, treating temperature, and reaction time. Therefore, this study focused on reaction termination processes, which have been ignored before. Interestingly, the natural cooling of Cd-based QDs is typically adopted during the reaction termi-

\footnotetext{
${ }^{1}$ MIIT Key Laboratory of Advanced Display Materials and Devices, Institute of Optoelectronics \& Nanomaterials, College of Materials Science and Engineering, Nanjing University of Science and Technology, Nanjing 210094, China

${ }^{2}$ Key Laboratory of Optoelectronic Technology \& Systems (Ministry of Education), Chongqing University, Chongqing 400044, China

${ }^{3}$ School of Microelectronics and Communication Engineering, Chongqing University, Chongqing 400044, China

${ }^{\dagger}$ These authors contributed equally to this work.

* Corresponding author (email: ywang@njust.edu.cn)
} 
nation process, while the ice water-cooling method results in a higher quality of perovskite-based QDs $[28,29]$. This indicates that the reaction termination stage or the cooling process may play an important role in improving the ZnSeTe QDs, which represent a new class of QD systems.

This paper reports for the first time the excited-state regulation in $\mathrm{ZnSeTe}$ core-shell QDs by a cooling-rate-controlling strategy. The presence of hot-carrier trapping $(<2 \mathrm{ps})$ and ultrafast band-edge carrier trapping $(\sim 5.5 \mathrm{ps})$ is responsible for the poor emission efficiency in ZnSeTe QDs. The above processes can be suppressed simultaneously by the cooling engineering, which strongly impacts the crystalline quality and surface/interface states, as revealed by the combined electronic and spectroscopic characterization. The cooling-optimized ZnSeTe QDs manifest a record-high PLQY of $>90 \%$ and outstanding stability, which are comparable to those of the canonical CdSe QDs. Leveraging the above achievement, the ecofriendly ZnSeTe QDs were used as emitters in white LEDs (WLEDs) in conjunction with red phosphors and blue chips. The WLEDs exhibit excellent optical performance, including a high color-rendering index (CRI) of 80 and an appropriate correlated color temperature (CCT) of $7391 \mathrm{~K}$, highlighting their prominent potential in solid-state illumination. Furthermore, the WLEDs are favorable light sources in eco-friendly visible light communication (e-VLC). With the modulation of orthogonal frequency division multiplexing (OFDM), the $-3 \mathrm{~dB}$ bandwidth and maximum achievable rate of e-VLC have reached $921 \mathrm{kHz}$ and $42.19 \mathrm{Mbps}$, respectively. These findings suggest that eco-friendly QDs could be feasible for practical applications.

\section{EXPERIMENTAL SECTION}

\section{Synthesis of multi-shelled ZnSeTe/ZnSe/ZnS QDs}

The synthesis was carried out using the classical heat injection method. The Se-diphenylphosphine (Se-DPP) precursor was prepared by dissolving $0.158 \mathrm{~g}(2 \mathrm{mmol})$ of Se in $1 \mathrm{~mL}$ of DPP at $100^{\circ} \mathrm{C}$; the concentration was $2 \mathrm{~mol} \mathrm{~L}^{-1}$. The Te-trioctyphosphine (Te-TOP) precursor was prepared by dissolving $0.089 \mathrm{~g}$ $(0.7 \mathrm{mmol})$ of Se in $3 \mathrm{~mL}$ of TOP under the same conditions. The concentration was $0.233 \mathrm{~mol} \mathrm{~L}^{-1}$. Two millimole of $\mathrm{Zn}$ acetate $\left(\mathrm{Zn}(\mathrm{OAc})_{2}\right)$ powder with $2 \mathrm{~mL}$ of oleic ocid $(\mathrm{OA})$ and $15 \mathrm{~mL}$ of octadecene (ODE) were placed into a three-neck flask. Inert gas was flowed at $120^{\circ} \mathrm{C}$ for $1 \mathrm{~h}$ to remove air from the bottle. Subsequently, $0.5 \mathrm{~mL}$ of Se-DPP and $1.2 \mathrm{~mL}$ of Te-TOP were injected sequentially and reacted initially at $210^{\circ} \mathrm{C}$ for $30 \mathrm{~min}$. The mixture reacted at $270^{\circ} \mathrm{C}$ for $1 \mathrm{~h}$ to form the alloyed $\mathrm{ZnSeTe}$. The alloyed $\mathrm{ZnSeTe}$ was very unstable. Therefore, a protective layer shell should be constructed. Subsequently, $3.2 \mathrm{~mL}$ of a $\mathrm{Zn}$ oleate $\left(\mathrm{Zn}(\mathrm{OA})_{2}\right)$ stock solution $(3 \mathrm{mmol}$ of $\mathrm{Zn}(\mathrm{OAc})_{2}$ in $2 \mathrm{~mL}$ of $\mathrm{OA}$ and $1.2 \mathrm{~mL}$ of ODE) and $1 \mathrm{~mL}$ of the Se-TOP solution $(1.25 \mathrm{mmol}$ of Se in $1 \mathrm{~mL}$ of TOP) were coinjected slowly into the $\mathrm{ZnSeTe}$ core-growth solution to form the $\mathrm{ZnSe}$ shell. For the outermost shell $\mathrm{ZnS}$, an equivalent amount of the $\mathrm{Zn}(\mathrm{OA})_{2}$ solution and $1 \mathrm{~mL}$ of S-TOP were added to the mixture described above. The shelling formation reactions were maintained at $270^{\circ} \mathrm{C}$ for $60 \mathrm{~min}$.

\section{Cooling engineering}

Different cooling rates were used to cool down the reaction solution. The experiment was divided into three groups of samples. Sample 1 was placed into ice water for rapid cooling; sample 2 was cooled to room temperature in air; sample 3 was kept in a furnace to achieve a slow cooling rate. The resulting multi-shelled ZnSeTe/ZnSe/ZnS QDs were purified repeatedly with mixed solvents of hexane and excess acetone with centrifugation.

\section{Material characterization}

The PL spectra were measured using a Varian Cary Eclipse instrument with the excitation wavelength set to $350 \mathrm{~nm}$, while the absorption spectra were measured with a Shimadzu UV3600. QY measurements were conducted using a QuantaurusQY Absolute yield spectrometer (C11347-11, Hamamatsu Photonics, Japan) with an excitation wavelength of $365 \mathrm{~nm}$. The reference sample was measured first. The sample concentration was then adjusted to $0.2-0.3 \mathrm{~mol} \mathrm{~L}^{-1}$ to assess the yield. The time-resolved fluorescence decay curves were measured using a pulsed diode laser (Edinburgh Instruments, EPL-375; pulse width: $60.3 \mathrm{ps}$ ) at a $5 \mathrm{MHz}$ repetition rate with a power density of $0.017 \mathrm{~W} \mathrm{~cm}^{-2}$ in the test system. Transmission electron microscopy (TEM, FEI Tecnai G20) was carried out using a $\mathrm{Cu}$ grid. The crystal structure was examined by X-ray diffraction (XRD, Bruker-AXS D8 Advance). The absolute PLQY was determined using a Quantaurus-QY Absolute photoluminescence quantum yield spectrometer (C11347-11, Hamamatsu Photonics, Japan). Energy-dispersive X-ray spectroscopy (EDS, COXEM EM-30N field emission environment scanning electron microscope) was performed for elemental analysis.

\section{RESULTS AND DISCUSSION}

Ice water cooling, natural air cooling, and furnace-controlled cooling were adopted to adjust the reaction termination or cooling rate. Fig. 1a displays photographs of the as-fabricated $\mathrm{ZnSeTe} / \mathrm{ZnSe} / \mathrm{ZnS}$ core-shell QDs by different cooling approaches under room light and ultraviolet (UV) irradiation, and its energy dispersive spectrum is shown in Fig. S1 and Table S1. The slow-cooling sample exhibited stronger emission under UV light than the rapid-cooling sample. Fig. 1b shows the corresponding cooling rates. For a fair comparison, the ZnSeTe core-shell QDs were synthesized under the same conditions (see details in the EXPERIMENTAL SECTION), except that the cooling rates were different. The absorption, PL, PLQY, and time-resolved PL measurements were performed to characterize the optical properties of the samples quantitatively. Fig. 1c shows the UVvisible (UV-Vis) absorption spectra of the three representative samples. The position of the exciton peak was obtained by taking the minimum value of the quadratic differential of the curve (Fig. S2), showing a redshift with the decreasing cooling rate. The PL intensity of the QDs (Fig. 1d) showed a nearly four-fold increase in the slow-cooling (furnace) samples compared with the rapid-cooling (ice water) one. A small decrease of the full width at half maximum from 47 to $44 \mathrm{~nm}$ was observed, which was attributed to the focusing effect [30]. The PL decay lifetime of the slow-cooling sample (25 ns) was three times longer than that of the rapid-cooling ( $8 \mathrm{~ns}$ ) counterpart. The cooling rate can be adjusted systematically using the furnace. Eventually, the ZnSeTe QDs with record-high PLQY of $\sim 90 \%$ were obtained at a cooling rate of $\sim 3 \mathrm{~K} \mathrm{~min}^{-1}$ (Fig. 1d and Fig. S3), which was much higher than those of the ice water (23\%)- and air (57\%)cooling samples. The cooling process represents an important stage to regulate the excited-state recombination and improve 

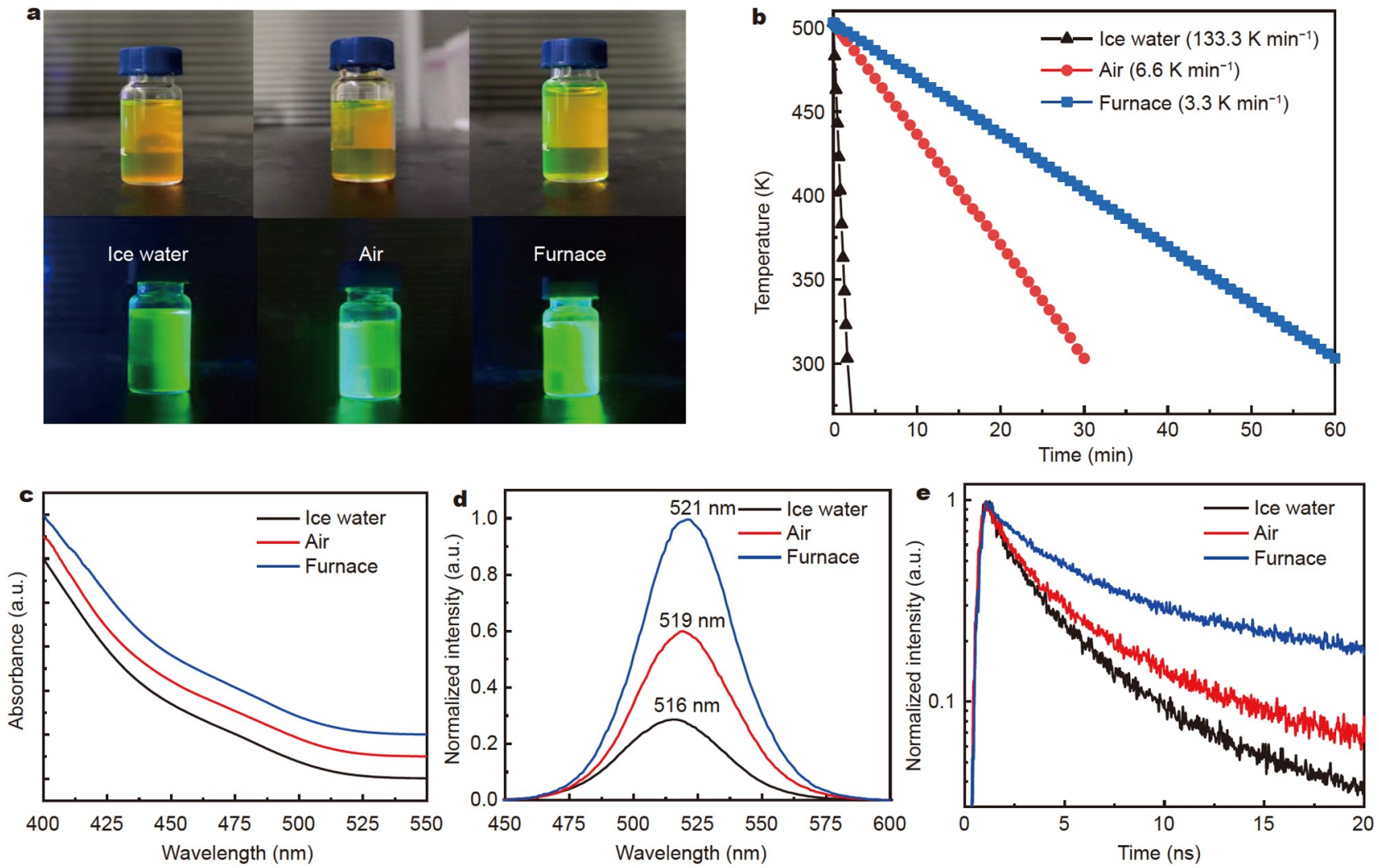

Figure 1 (a) Three QD solutions with different cooling rates under room light (top) and UV irradiation (bottom). Cooling temperature vs. time curves (b), UV absorption spectra (c), PL spectra (d), and fluorescence lifetimes (e) of the three samples with different cooling rates.

the quality of ZnSeTe-based QDs.

The transient absorption (TA) measurements with a time resolution of $\sim 100 \mathrm{fs}$ were performed to gain more insight (see details in the EXPERIMENTAL SECTION). The ZnSeTe QDs obtained by ice-water cooling, natural air cooling, and furnace cooling were excited by a relatively low pump energy of $31.8 \mu \mathrm{J} \mathrm{cm}^{-2}$ to avoid the Auger effect. Fig. $2 \mathrm{a}$ and b show the typical pseudo-color TA spectra of the rapid-cooling and slowcooling samples (see Fig. S4 in the Supplementary information for the other sample). A broad and remarkable feature of photobleaching $(\mathrm{PB})$ is observed, corresponding to the recombination dynamics of band-edge excitons [31,32] because it matches the steady-state absorption spectrum. Fig. $2 \mathrm{c}$ shows the TA spectral distribution at different delay times. By tracking the dynamics of the $\mathrm{PB}$ signal at the band-edge exciton, the photo-bleach kinetics at different cooling rates could be extracted, as shown in Fig. 2d. These decay curves showed remarkable differences, especially at the initial decay stage within $50 \mathrm{ps}$ (see the zoomed curves in Fig. S5). In detail, the rapid-cooling sample exhibited a prominent ultrafast decay process within the initial $50 \mathrm{ps}$, whereas this process almost disappeared in the slow-cooling sample (Fig. 2d). A three-exponential decay function was used to fit the bleaching recovery curve and extract the processes quantitatively (Table S2). A fast decay component of $\sim 5.5$ ps was observed in the rapid-cooling sample, indicating the existence of carrier defects in the band-edge. By changing the cooling rate, the defect-trapping component of the material was reduced from $28 \%$ to $<10 \%$. The suppression of ultrafast picosecond carrier trapping in ZnSeTe QDs by the cooling engineering will mitigate the nonradiative recombination and improve the emission performance. PL excitation (PLE) spectroscopy was carried out on the samples (Fig. 2e) to track the hot-carrier behavior [33,34]. The PLE spectrum begins to deviate from the absorption spectrum in a wavelength range shorter than $\sim 490 \mathrm{~nm}$ for the ice water-cooling sample, suggesting the significant hot-carrier trapping upon short-wavelength excitation in these samples. In contrast, the furnace-cooling sample showed suppressed hotcarrier trapping behavior, as manifested from the PLE/absorption spectrum, where the PLE begins to deviate from the absorption spectrum for much shorter wavelengths $(\sim 450 \mathrm{~nm})$. In addition, the deviation amplitude between the PLE and absorption spectrum was much smaller at a similar excitation wavelength for the furnace-cooling sample than ice water-cooling ones, further confirming the suppressed hot-carrier trapping in the furnace-cooling sample. To derive the hot-carrier trapping dynamics, the hot-carrier relaxation process was also investigated by monitoring the built-up kinetics of the band-edge carrier population, i.e., PB signal [32]. The hot-carrier underwent relaxation within $\sim 1$ ps (Fig. 2f), indicating the ultrafast hot-carrier trapping process in $\mathrm{ZnSeTe}$ QDs. These results suggest that besides band-edge carrier trapping, hot-carrier trapping is also important for $\mathrm{ZnSeTe}$ QDs, whereas the cooling strategy plays a role in suppressing band-edge carrier trapping and mitigating the hot-carrier trapping.

TEM and XRD were conducted to determine the changes in ZnSeTe QDs during the cooling process. TEM showed that the size of the prepared QDs increased gradually with the decreasing cooling rate (Fig. 3a and Fig. S6). During the cooling process, 

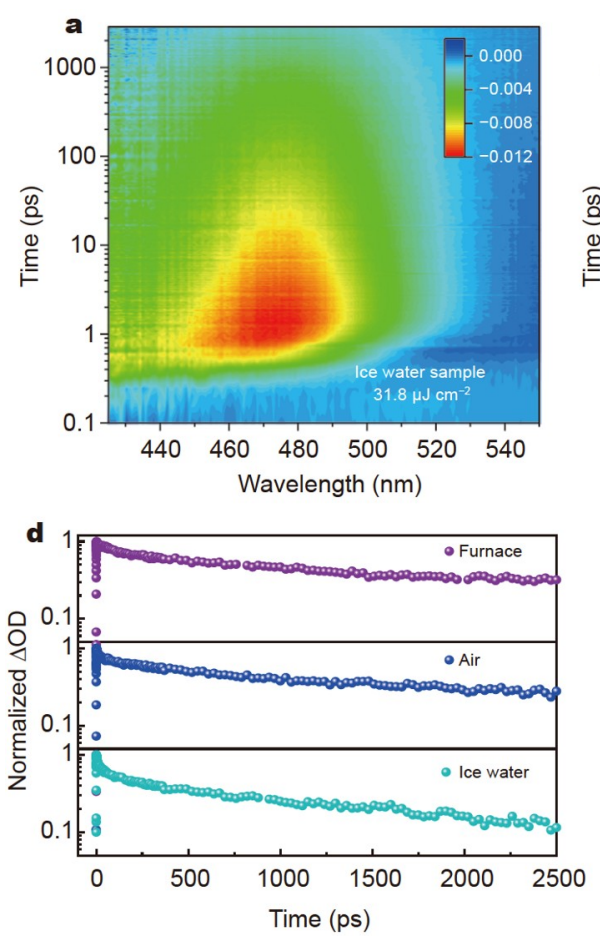
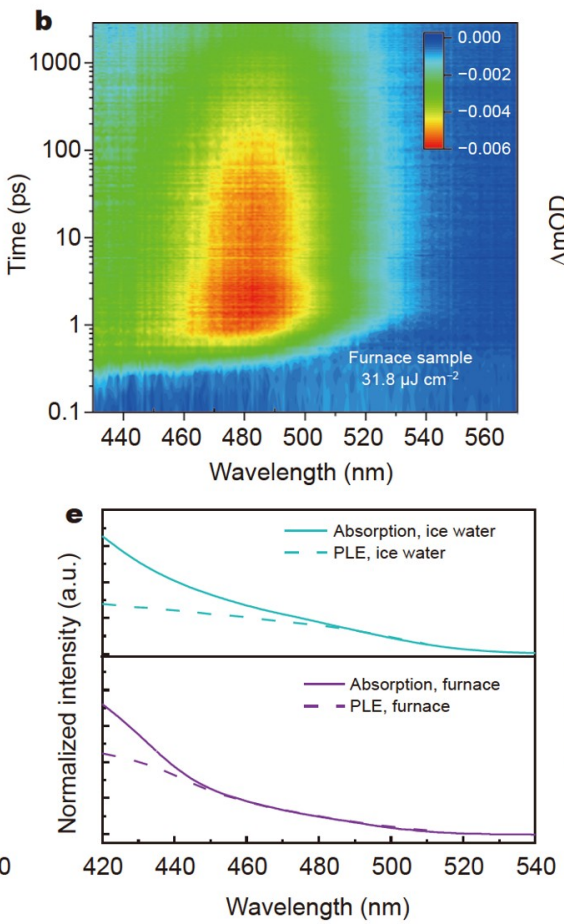
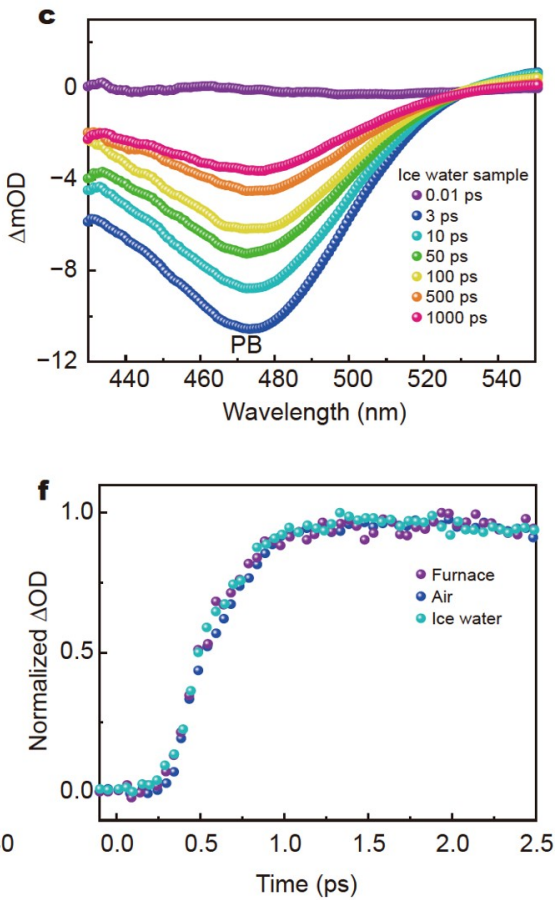

Figure 2 Pseudo-color TA spectra of the ice water-cooling sample (a) and furnace-cooling sample (b). (c) The corresponding TA spectra of the ice watercooling sample extracted at different delay times. (d) Recovery kinetics of photo-bleaching peaks. (e) PLE and absorption spectra of the ice water-cooling sample and furnace-cooling sample. (f) The normalized rising curves of the three samples probed at the band edge.

supersaturation of the monomers occurs, which drives the growth of the QDs (see the corresponding LaMer growth model in Fig. S7). As mentioned in the EXPERIMENTAL SECTION, the reaction time of the inner ZnSe shell was longer during the shelling process. Therefore, there are more $\mathrm{ZnS}$ monomers than $\mathrm{ZnSe}$ in the solution, and the re-growth during cooling mainly arises from the thickening of the $\mathrm{ZnS}$ shell. Moreover, the growth of QDs requires contact with monomers in the reaction solution, while this process can be blocked by surfactant molecules containing both metal-coordinating groups and solvophilic groups [35]. The dynamic exchange between surfactant and monomers requires a certain temperature. Therefore, the QDs with a slow cooling rate could have more time for shell growth than the rapid-cooling samples and become larger (Fig. S8). Importantly, as manifested by the TEM images, the slow-cooling samples showed improved crystalline quality, where the lattice structure can be clearly observed (Fig. 3b) and that it is absent for the rapid-cooling sample. The higher crystallinity quality of the QDs would enable fewer lattice defects and was beneficial for a high PLQY.

The thicker shell may better passivate the surface states and render a smaller overlap between the wave functions of the photoexcited carriers and surface states [36,37]. Therefore, a suppressed nonradiative recombination induced by surface traps is anticipated, which gives rise to a high PLQY. However, with the increasing shell thickness, dislocations would be formed at the interface between the layers to offset the strain required for lattice matching [38]. In this case, interface defects would be formed for thick-shell samples if any lattice mismatch cannot be counterbalanced, leading to deteriorated PLQY. This is certainly not the case in ZnSeTe QDs. During the furnace-controlled slow-cooling process, atomic diffusion may occur in the $\mathrm{ZnSeTe}$ core-shell QDs to offset the lattice mismatch because it commonly occurs for the ternary and quaternary alloys [39]. Fig. 3c shows the XRD patterns of the three samples along with the standard XRD patterns of ZnTe, ZnSe, and ZnS bulk crystals (vertical solid lines). The smooth region at the peak corresponds to the mixed peaks of $\mathrm{ZnSe}_{1-x} \mathrm{Te}_{x}, \mathrm{ZnSe}$, and $\mathrm{ZnSe}_{1-x} \mathrm{~S}_{x}$. According to Fig. $3 c$, the slower cooling process will produce more $\mathrm{ZnSeS}$ with different proportions, suggesting that the diffusion between different layers is more prominent. Hence, the slow-cooling method retains the QDs at high temperatures for a sufficient time to form a wider alloyed interface by interatomic diffusion. The transformation region between the core and shell becomes smooth (Fig. 3d), ensuring a thick shell without generating interface defects and contributing to the high PLQY.

Moreover, it might exhibit low-temperature growth during the slow-cooling method. In this process, the growth of the $\mathrm{ZnS}$ monomer eventually produces a metastable shell with a smooth shell surface when the temperature decreases due to insufficient energy to overcome the smooth to rough activation barrier [40]. Such metastable shells could effectively suppress the defect states while maintaining high lattice strain energy. The slow-cooling method makes this process possible because of the longer time in the same temperature range, and the $\mathrm{ZnS}$ monomer maintains dynamic equilibrium during the slow cooling [37].

In addition to the high PLQY, the thick-shell QDs by the slowcooling method may also help enhance the stability. The ZnSeTe core-shell QDs with the slow-cooling process show almost no detectable change after storage for at least 3 months (Fig. S9). For a strict comparison, the stability of the as-prepared $\mathrm{ZnSeTe}$ core-shell QDs under intense UV irradiation was studied and compared with the classical CdSe/CdS/ZnS QDs, which have proven qualified stability toward practical applications [41]. 

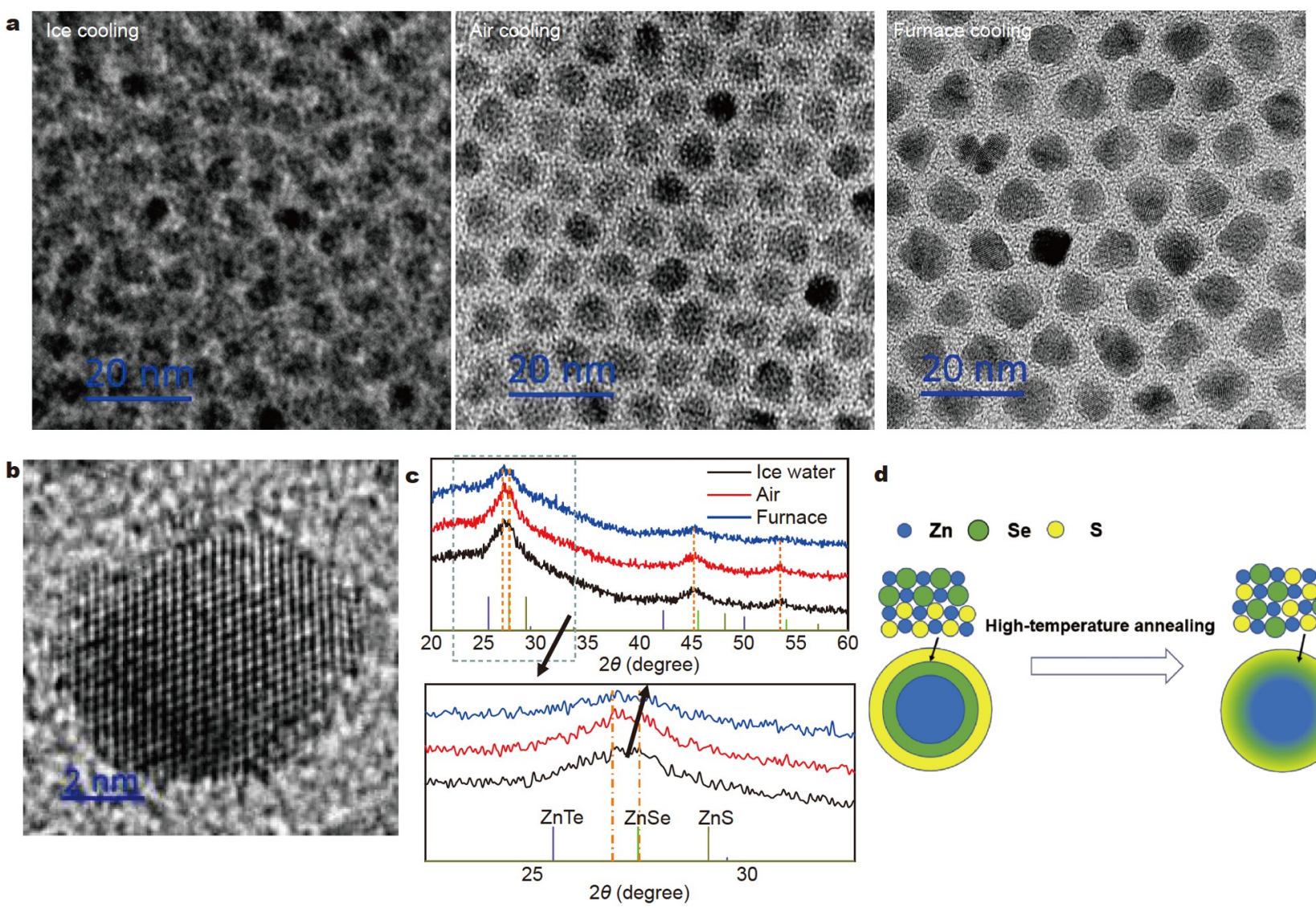

d
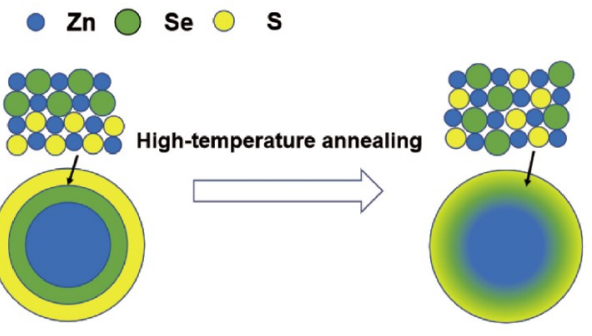

Figure 3 (a) TEM images with different cooling methods. (b) High-resolution TEM of ZnSeTe/ZnSe/ZnS lattice structure by the slow-cooling method. (c) XRD patterns of three QDs with different cooling rates. The bottom is the first diffraction peak under local magnification of $2 \theta=25^{\circ}-30^{\circ}$. (d) Model of the diffusion of $\mathrm{S}$ atoms from the $\mathrm{ZnS}$ layer to the ZnSe layer.

In the experiment, the samples were exposed to a high-power $\left(\sim 300 \mathrm{~mW} \mathrm{~cm}^{-2}\right)$ UV lamp to assess the light stability. The $\mathrm{ZnSeTe}$ core-shell QDs with a fast cooling rate showed a rapidly decreased PL intensity under long-term UV irradiation (Fig. 4c). It was almost quenched under less than $10 \mathrm{~h}$ of irradiation. This was attributed to the generation of photoinduced defects for the rapid-cooling sample due to incomplete external shell coating. On the other hand, the slow-cooling sample showed improved stability. As shown in Fig. $4 \mathrm{a}$, after $10 \mathrm{~h}$ of continuous irradiation, the PL of the sample was quenched only by $\sim 30 \%$.

The light stability of $\mathrm{CdSe} / \mathrm{CdS} / \mathrm{ZnS}$ was also tested as a comparative experiment in Fig. 4b. The PL intensity decreased approximately $40 \%$ under similar UV irradiation. Fig. $4 \mathrm{c}$ shows the PL intensity variation of the samples as a function of the UV irradiation time. Overall, the optimized nontoxic QDs showed improved stability, comparable to the CdSe/CdS/ZnS QDs. This observation is conducive to practical optoelectronic applications.

The effective slow-cooling strategy is universal for $\mathrm{ZnSeTe}$ QDs with varying compositions or emission colors. Three lightemitting QDs were synthesized by setting the Te/Se ratios to $0.07,0.14$, and 0.28 , respectively. The fabrication procedure was similar to those of the green-emitting QDs; the only difference was the ratio of the precursors, which is controlled by the amounts of Te and Se. Fig. 4d shows a photograph of a ZnSeTe core-shell QD solution with different emission colors fabricated using the optimized cooling process. All the samples exhibited favorably high PLQY, where the blue emitters reached a PLQY of $\sim 95 \%$, and the yellow-green emitters yielded $\sim 90 \%$. Fig. $4 \mathrm{e}$ shows the corresponding PL spectra, where the emission wavelengths are 470,500 , and $520 \mathrm{~nm}$, respectively. This suggests that the emission can be adjusted easily from blue-to-green for efficient luminescence. In addition, Fig. $4 \mathrm{f}$ shows the time-resolved PL spectra of the samples. The lifetimes were relatively long in the range of tens of nanoseconds. The slightly increased PL lifetime for shorter wavelength emission may be due to the intrinsic optical transition properties of the ZnSeTe QDs.

As a proof of concept, the optimized nontoxic QDs were used for white light emission and VLC. The ZnSeTe core-shell QDs and red-emitting phosphors were dropped onto blue chips to fabricate the WLEDs. As shown in Fig. $5 \mathrm{a}$, when driven at $2.5 \mathrm{~V}$, the WLEDs emitted a broad spectrum with three peaks, corresponding to the luminescence of the green $\mathrm{ZnSeTe} Q \mathrm{QDs}$, red phosphors, and blue chips, respectively. The inset in Fig. 5a shows a photograph of an operating WLED, demonstrating its bright white emission in the dark. Moreover, the WLEDs exhibit Commission Internationale de L'Eclairage (CIE) coordinates of $(0.30,0.33$ ), a high CRI of 80 (Fig. 5b) and a proper CCT of $7391 \mathrm{~K}$, suggesting their promising applications in solid-state illumination. In addition, considering the potential of QDs toward a high modulation bandwidth and high data transmission rates in light communication systems, the WLEDs could be used as a light source in wireless VLC application. Fig. $6 a$ and $b$ display the obtained electrical-optical-electrical (EOE) frequency response and received signal-to-noise (SNR) of the VLC 

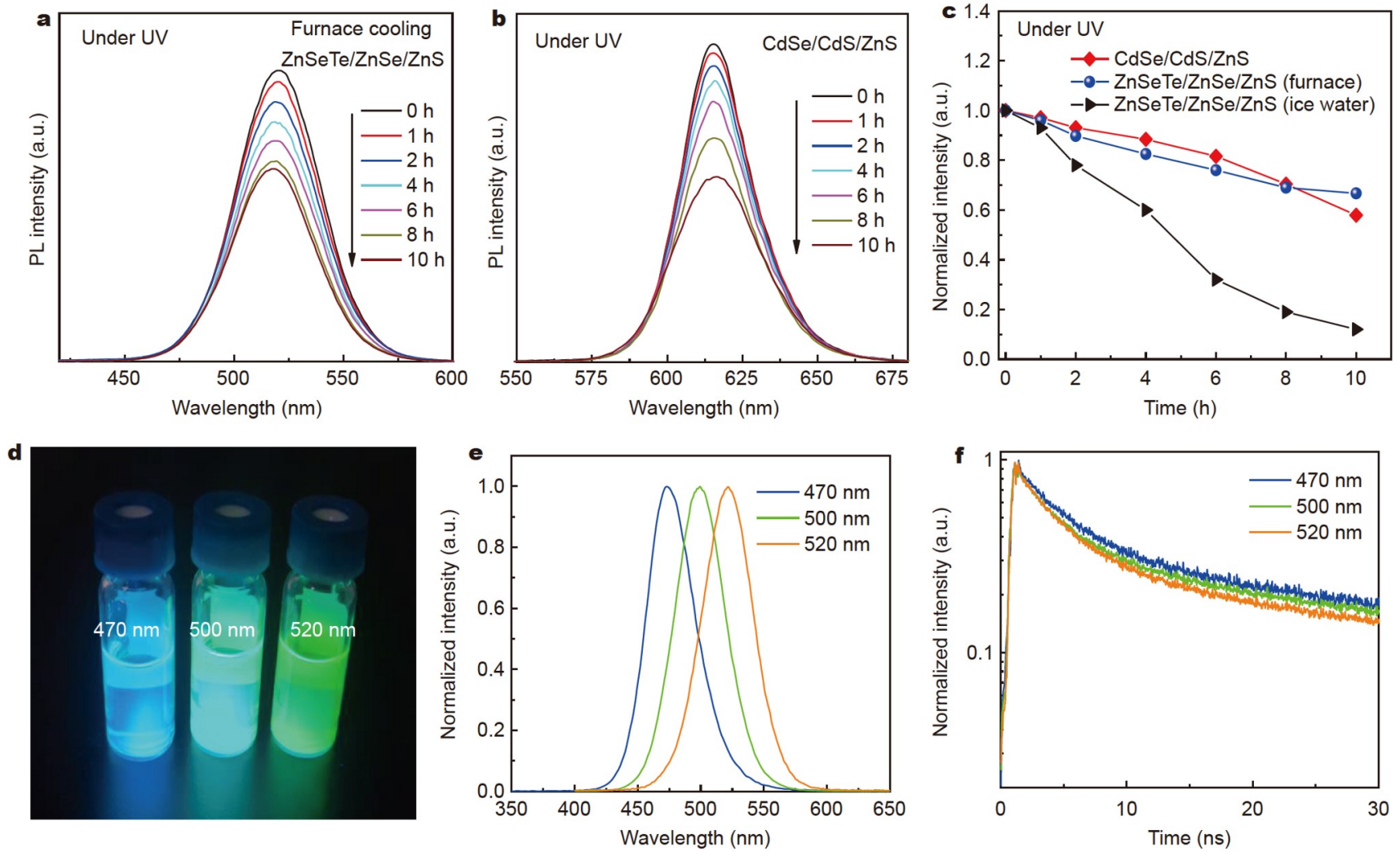

Figure 4 (a) PL spectra of ZnSeTe/ZnSe/ZnS and (b) CdSe/CdS/ZnS under UV irradiation. (c) Normalized PL intensities of CdSe/ZnSe/ZnS and ZnSeTe/ $\mathrm{ZnSe} / \mathrm{ZnS}$ with different cooling rates under UV irradiation. Photographs (d), PL spectra (e) and fluorescence lifetimes (f) of the samples with different emission wavelengths.
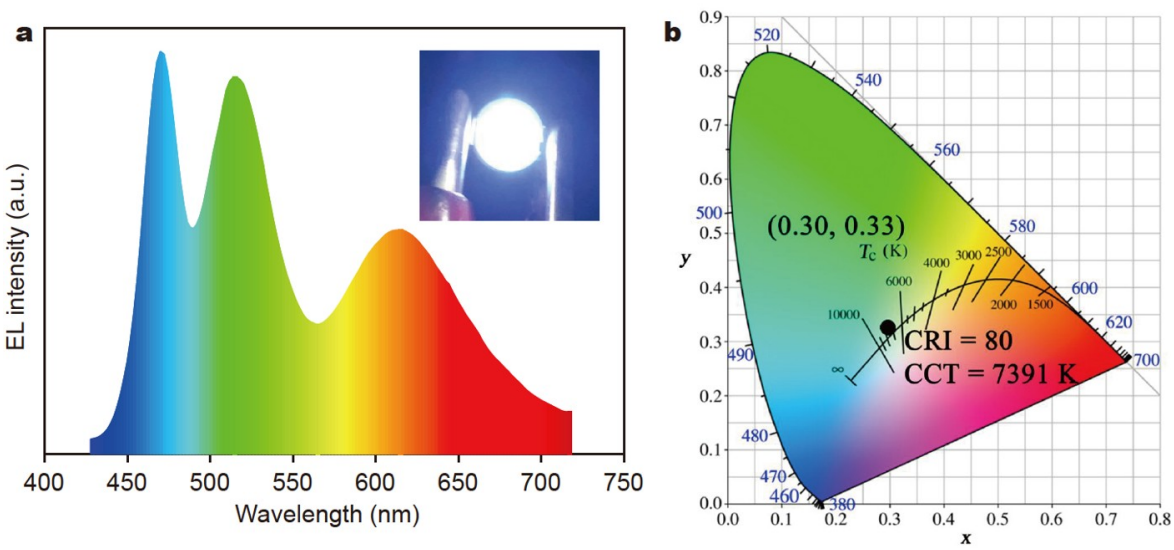

Figure 5 (a) EL spectrum of the as-fabricated WLEDs. The inset is the photograph of a working WLED in the dark. (b) CIE chromaticity diagram of the WLEDs.

system, respectively. The EOE result shows a $-3 \mathrm{~dB}$ bandwidth of $921 \mathrm{kHz}$ for the VLC system, indicating typical low-pass characteristics. Moreover, the SNR decreased gradually with increasing electrical frequency but maintained a high value to distinguish the signals. To illustrate the signal transmitting ability of the VLC systems further, the WLEDs were modulated by a typical square wave with a frequency of $1 \mathrm{MHz}$. Fig. $6 \mathrm{c}$ shows the corresponding eye diagram, which manifests that the WLEDs can transmit the optical signal under the frequency of $1 \mathrm{MHz}$. Furthermore, by applying an OFDM with a bit loading, the WLEDs demonstrate a maximum bit loading of
5 bits $\mathrm{s}^{-1} \mathrm{~Hz}^{-1}$ (Fig. 6d). Fig. 6e presents the constellation diagrams of the bit loading received, including binary phase shift keying (BPSK), 4-ary quadrature amplitude modulation (4 QAM), 8 QAM, 16 QAM, and 32 QAM. The transmitting data rate of the VLC system was $42.19 \mathrm{MHz}$, which was more than 45 times that of the obtained bandwidth.

\section{CONCLUSIONS}

This paper reported a novel cooling-rate-controlling method to grow high-quality ZnSeTe QDs with high PLQY (up to 90\%) and outstanding stability comparable to the canonical $\mathrm{CdSe} /$ 

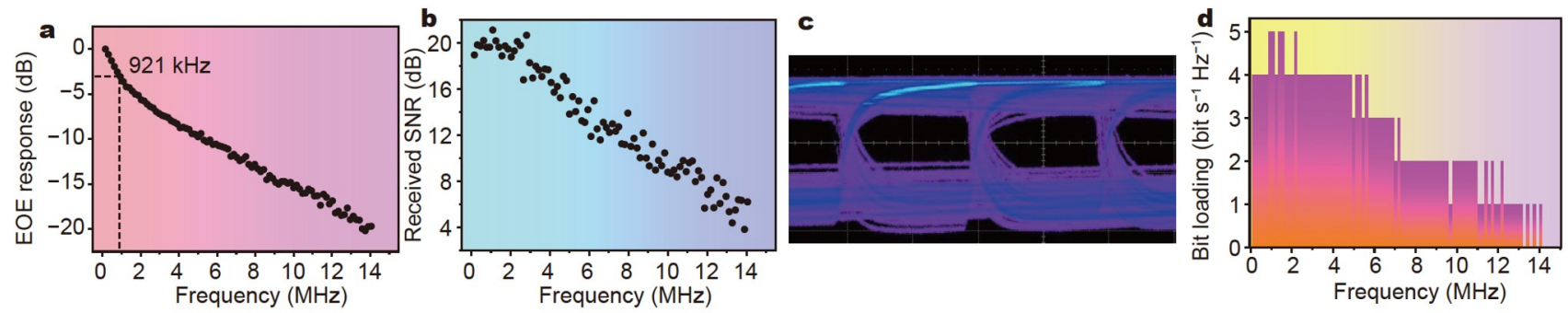

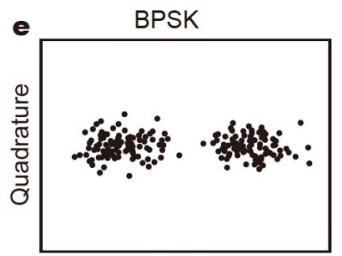

In-phase

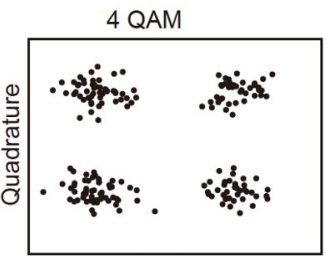

In-phase

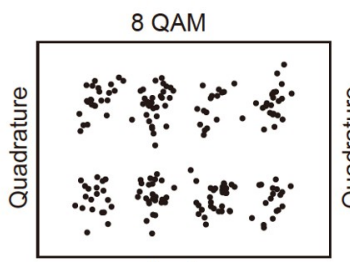

In-phase

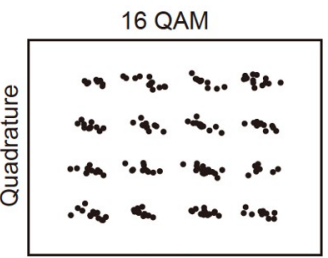

In-phase

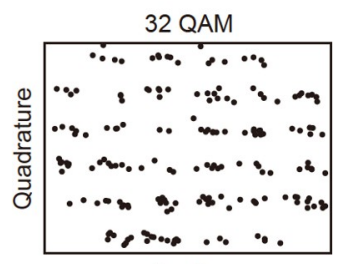

In-phase

Figure 6 (a) EOE frequency response and (b) received frequency SNR as a function of the electrical frequency in the VLC system. (c) Eye diagram and (d) bit loading profile of the VLC system. (e) Constellation diagram of BPSK, 4 QAM, 8 QAM, 16 QAM, and 32 QAM.

CdS/ZnS QDs. The cooling process strongly influenced the crystalline quality, shell thickness, and the surface/interface defects of ZnSeTe-based QDs through comprehensive electronic and spectroscopic analysis. WLEDs were fabricated by combining the as-prepared ZnSeTe QDs and red phosphors on blue chips. Finally, the WLEDs were used as a favorable light source in VLC and showed excellent potential for optical communication.

Received 2 November 2021; accepted 4 January 2022; published online 11 February 2022

1 Shirasaki Y, Supran GJ, Bawendi MG, et al. Emergence of colloidal quantum-dot light-emitting technologies. Nat Photon, 2012, 7: 13-23

2 Yoon SY, Kim JH, Kim KH, et al. High-efficiency blue and white electroluminescent devices based on non-Cd I-III-VI quantum dots. Nano Energy, 2019, 63: 103869

3 Won YH, Cho O, Kim T, et al. Highly efficient and stable InP/ZnSe/ ZnS quantum dot light-emitting diodes. Nature, 2019, 575: 634-638

4 Dai X, Zhang Z, Jin Y, et al. Solution-processed, high-performance light-emitting diodes based on quantum dots. Nature, 2014, 515: 96-99

5 Wang A, Shen $\mathrm{H}$, Zang S, et al. Bright, efficient, and color-stable violet ZnSe-based quantum dot light-emitting diodes. Nanoscale, 2015, 7: 2951-2959

6 Luo Z, Chen Y, Wu ST. Wide color gamut LCD with a quantum dot backlight. Opt Express, 2013, 21: 26269-26284

7 Padilha LA, Nootz G, Olszak PD, et al. Optimization of band structure and quantum-size-effect tuning for two-photon absorption enhancement in quantum dots. Nano Lett, 2011, 11: 1227-1231

8 Regulacio MD, Han MY. Composition-tunable alloyed semiconductor nanocrystals. Acc Chem Res, 2010, 43: 621-630

9 Zhang C, Chen J, Kong L, et al. Core/shell metal halide perovskite nanocrystals for optoelectronic applications. Adv Funct Mater, 2021, 31: 2100438

10 Qu L, Peng ZA, Peng X. Alternative routes toward high quality CdSe nanocrystals. Nano Lett, 2001, 1: 333-337

$11 \mathrm{Lu} \mathrm{M}$, Zhang Y, Wang S, et al. Metal halide perovskite light-emitting devices: Promising technology for next-generation displays. Adv Funct Mater, 2019, 29: 1902008

12 Lin K, Xing J, Quan LN, et al. Perovskite light-emitting diodes with external quantum efficiency exceeding 20 percent. Nature, 2018, 562: 245-248

13 Xie R, Battaglia D, Peng X. Colloidal InP nanocrystals as efficient emitters covering blue to near-infrared. J Am Chem Soc, 2007, 129:

\section{$15432-15433$}

14 Li L, Reiss P. One-pot synthesis of highly luminescent InP/ZnS nanocrystals without precursor injection. J Am Chem Soc, 2008, 130: 1158811589

$15 \mathrm{Gu} \mathrm{YP}$, Cui R, Zhang ZL, et al. Ultrasmall near-infrared $\mathrm{Ag}_{2} \mathrm{Se}$ quantum dots with tunable fluorescence for in vivo imaging. J Am Chem Soc, 2012, 134: 79-82

16 Langevin MA, Ritcey AM, Allen CN. Air-stable near-infrared AgInSe nanocrystals. ACS Nano, 2014, 8: 3476-3482

17 Zhang W, Ding S, Zhuang W, et al. InP/ZnS/ZnS core/shell blue quantum dots for efficient light-emitting diodes. Adv Funct Mater, 2020, 30: 2005303

18 Asano H, Arai K, Kita M, et al. Synthesis of colloidal Zn(Te,Se) alloy quantum dots. Mater Res Express, 2017, 4: 106501

19 Li C, Nishikawa K, Ando M, et al. Synthesis of Cd-free water-soluble $\mathrm{ZnSe}_{1-x} \mathrm{Te}_{x}$ nanocrystals with high luminescence in the blue region. J Colloid Interface Sci, 2008, 321: 468-476

20 Jang EP, Han CY, Lim SW, et al. Synthesis of alloyed ZnSeTe quantum dots as bright, color-pure blue emitters. ACS Appl Mater Interfaces, 2019, 11: 46062-46069

21 Han CY, Lee SH, Song SW, et al. More than 9\% efficient $\mathrm{ZnSeTe}$ quantum dot-based blue electroluminescent devices. ACS Energy Lett, 2020, 5: 1568-1576

22 Lee SH, Han CY, Song SW, et al. ZnSeTe quantum dots as an alternative to InP and their high-efficiency electroluminescence. Chem Mater, 2020, 32: 5768-5775

23 Peng ZA, Peng X. Formation of high-quality CdTe, CdSe, and CdS nanocrystals using CdO as precursor. J Am Chem Soc, 2001, 123: 183184

24 Peng X, Manna L, Yang W, et al. Shape control of CdSe nanocrystals. Nature, 2000, 404: 59-61

25 Reiss $\mathrm{P}$, Carrière $\mathrm{M}$, Lincheneau $\mathrm{C}$, et al. Synthesis of semiconductor nanocrystals, focusing on nontoxic and earth-abundant materials. Chem Rev, 2016, 116: 10731-10819

26 Abe S, Čapek RK, De Geyter B, et al. Tuning the postfocused size of colloidal nanocrystals by the reaction rate: From theory to application. ACS Nano, 2012, 6: 42-53

27 Sugimoto T. Preparation of monodispersed colloidal particles. Adv Colloid Interface Sci, 1987, 28: 65-108

28 Luo C, Yan C, Li W, et al. Ultrafast thermodynamic control for stable and efficient mixed halide perovskite nanocrystals. Adv Funct Mater, 2020, 30: 2000026

29 Dabbousi BO, Rodriguez-Viejo J, Mikulec FV, et al. (CdSe)ZnS core -shell quantum dots: Synthesis and characterization of a size series of highly luminescent nanocrystallites. J Phys Chem B, 1997, 101: 9463- 


\section{5}

Peng X, Wickham J, Alivisatos AP. Kinetics of II-VI and III-V colloidal semiconductor nanocrystal growth: "Focusing" of size distributions. J Am Chem Soc, 1998, 120: 5343-5344

31 Wei $\mathrm{K}$, Zheng $\mathrm{X}$, Cheng $\mathrm{X}$, et al. Observation of ultrafast excitonexciton annihilation in $\mathrm{CsPbBr}_{3}$ quantum dots. Adv Opt Mater, 2016, 4: 1993-1997

32 Ren Y, Nie Z, Deng F, et al. Deciphering the excited-state dynamics and multicarrier interactions in perovskite core-shell type hetero-nanocrystals. Nanoscale, 2021, 13: 292-299

33 Kambhampati P. Hot exciton relaxation dynamics in semiconductor quantum dots: Radiationless transitions on the nanoscale. J Phys Chem C, 2011, 115: 22089-22109

34 Felici M, Polimeni A, Miriametro A, et al. Free carrier and/or exciton trapping by nitrogen pairs in dilute $\mathrm{GaP}_{1-x} \mathrm{~N}_{x}$. Phys Rev B, 2005, 71: 045209

35 Yin Y, Alivisatos AP. Colloidal nanocrystal synthesis and the organicinorganic interface. Nature, 2005, 437: 664-670

$36 \mathrm{Fu} \mathrm{Y}$, Kim D, Jiang W, et al. Excellent stability of thicker shell CdSe@ZnS/ZnS quantum dots. RSC Adv, 2017, 7: 40866-40872

37 Pal BN, Ghosh Y, Brovelli S, et al. 'Giant' CdSe/CdS core/shell nanocrystal quantum dots as efficient electroluminescent materials: Strong influence of shell thickness on light-emitting diode performance. Nano Lett, 2012, 12: 331-336

38 Song KK, Lee S. Highly luminescent (ZnSe)ZnS core-shell quantum dots for blue to UV emission: synthesis and characterization. Curr Appl Phys, 2001, 1: 169-173

39 Lin GR, Lo TC, Tsai LH, et al. Finite silicon atom diffusion induced size limitation on self-assembled silicon quantum dots in silicon-rich silicon carbide. J Electrochem Soc, 2011, 159: K35-K41

40 Nan W, Niu Y, Qin H, et al. Crystal structure control of zinc-blende CdSe/CdS core/shell nanocrystals: Synthesis and structure-dependent optical properties. J Am Chem Soc, 2012, 134: 19685-19693

41 Wang Y, Ta VD, Gao Y, et al. Stimulated emission and lasing from $\mathrm{CdSe} / \mathrm{CdS} / \mathrm{ZnS}$ core-multi-shell quantum dots by simultaneous threephoton absorption. Adv Mater, 2014, 26: 2954-2961

Acknowledgements This work was supported by the National Natural Science Foundation of China (11904172) and Natural Science Foundation of Jiangsu Province (BK20190446). Wang Y thanks the support of the start-up funding from Nanjing University of Science and Technology.

Author contributions Wang $\mathrm{Y}$ conceived the idea; Wu B prepared the materials; Wu B, Zhang $\mathrm{M}$, and Huang $\mathrm{Z}$ conducted the characterization of materials. All the authors commented on the manuscript.

Conflict of interest The authors declare that they have no conflict of interest.

Supplementary information Supporting data are available in the online version of the paper.

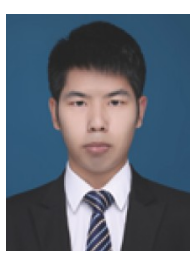

Baoqiang Wu received his B.S. degree from Wuhan University of Science and Technology in 2019. He is now studying for a master's degree in the School of Materials Science and Engineering at Nanjing University of Science and technology. His main research is the preparation and application of cadmium-free semiconductor nanocrystals.

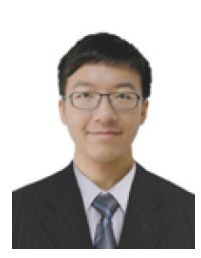

Shuangyi Zhao received his B.S. degree from Shandong University in 2013 and Ph.D. degree from the State Key Lab of Silicon Materials at Zhejiang University in 2018. He is now a postdoctoral researcher in the College of Optoelectronic Engineering at Chongqing University. His research concerns the fabrication of semiconductor nanocrystals, including lead-halide and lead-free perovskites, and their applications in optoelectronic devices.

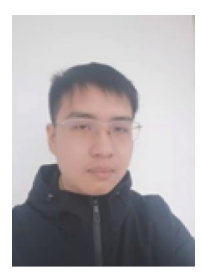

Mingshui Zhang received his B.S. degree at Nanjing University of Science and Technology in 2020. He is now studying for a master's degree in the School of Materials Science and Engineering at Nanjing University of Science and Technology. His main research is the preparation and application of perovskite core-shell nanocrystals.

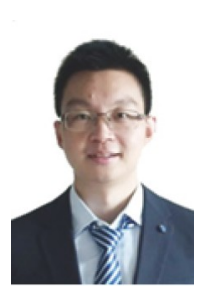

Yue Wang received his Ph.D. degree in physics from Nanyang Technological University. He is currently working at the School of Materials Science and Engineering, Nanjing University of Science and Technology. His research interests include optical spectroscopy, semiconductor photophysics, and optically pumped lasers.

\section{基于冷却工程对环保型 $\mathrm{ZnSeTe}$ 基量子点的激发态 调控}

吴保强 ${ }^{1 \dagger}$, 赵双易 ${ }^{2 \dagger}$, 张明水 ${ }^{1 \dagger}$, 黄志高 ${ }^{1}$, 陈晨 $^{3}$, 藏志刚 ${ }^{2}$, 王跃 ${ }^{1 *}$

摘要 由于材料内部存在高效的非辐射复合, 这使得实现高质量的环 保量子点仍然具有挑战性. 本文中, 我们探索了通过冷却工程调节激发 态对实现高质量的ZnSeTe核壳量子点的影响. 研究发现, 超快热载流子 俘获和带边载流子俘获是 $\mathrm{ZnSeTe}$ 量子点发射效率低下的主要原因. 我 们通过设计冷却工程抑制上述过程, 并且结合电子和光谱表征分析了 其潜在机制. 经过冷却优化的 $\mathrm{ZnSeTe}$ 量子点表现出高量子发光效率 (>90\%)和稳定性，这可与经典的 CdSe量子点相媲美. 基于这种量子点 的白光发光二极管 (WLED) 表现出优异的光学性能, 包括高显色指数 (80)和良好的相关色温 $(7391 \mathrm{~K})$. 此外, 高性能的WLED还可以用作环 保可见光通信. 这些结果表明了环保量子点在实际应用中的可行性. 